\title{
The co-morbidity of PTSD and substance abuse:A military case review and general considerations
}

\section{Opinion}

I served as the psychiatrist for the Defence and Veterans Brain Injury Center, a national model program that treated veterans of the Iraq and Afghanistan wars who had traumatic brain injuries and were suffering from post-traumatic stress disorder. The soldiers were sent to our program only after failing to respond to military hospital treatment for these conditions for at least a year. I saw multiple patients who showed the strong link between developing substance abuse co-morbid with pre-existing post-traumatic stress disorder. The severity of these soldiers' war experiences was often overwhelming, and their substance use was one of much behaviour that led them to a rapid decomposition from their previous high level of functioning.

The following soldier's case is representative of what I saw in many of these chronically ill veterans.

The twenty-five-year-old man had entered the service at the age of nineteen. He was sent to Iraq, where he was the private at a border crossing, acting as the sentry. Two young eight-year-old boys walked toward the border crossing, and they did not stop when they were told in Arabic to do soand when shots were fired over their heads. The commanding sergeant at the border crossing told the novice soldier to shoot the boys. The private turned to the sergeant and said he would not shoot children. The sergeant's immediate response: "I'll shoot them if you don't, and you'll be put on trial for disobeying your commanding officer."

The private shot the two boys. They both had mobile explosiveladen devices and had been told exactly where to go and not to stop. If they had not been shot and had gotten closer to the border crossing, multiple U.S. soldiers would have been killed.

The private returned home and was unable to sleep. He had nightmares of children he had to kill as soon as he fell asleep. His irritability increased, he broke up with his girlfriend after hitting her, he lost his job, and he began to drink heavily. He then turned to pain pills along with the alcohol to try to numb himself. He became homeless and unable to afford the pain pills. Finally, three years after his return from combat, he robbed a bank with a handgun to obtain money for heroin.

The private was caught and was put in jail. A senior judge hearing his story knew he was suffering from PTSD, and he was put in our program. I hospitalized the soldier, and he was given large doses of sedatives to force sleep. He would only sleep, eat, and be put back to sleep. After over a week of this treatment, his thinking cleared, he was able to interact and not be completely withdrawn. I recommended to the judge that the soldier required ongoing treatment in our program. The judge deferred sentencing and allowed continued intensive treatment while also allowing him to return to his family's farm,
Volume 5 Issue 6 - 2018

\author{
Burton Singerman \\ Psychiatrist in Pittsburgh, USA
}

Correspondence: Burton Singerman, psychiatrist in Pittsburgh, USA, Email burtseng@gmail.com

Received: December 07, 2017 | Published: November 20, 2018

where he grew up. He tended to the animals and made progress over two years with both pharmacologic and psychotherapeutic treatment, reliving his experiences and dealing with his guilt.

Two years after his arrest, he was given a sentence for his armed robbery, with leniency shown because of his history and his progress. His early life history had no risk factors for either substance abuse or antisocial acts. Clearly, his severe PTSD and chronic sleep deprivation led him to his unlawful actions. There were no military courts to evaluate the role of his PTSD at this early time. A senior judge created a truly humane sentence based on the understanding of his psychopathology.

Post-traumatic stress is highly linked with substance abuse in both veteran and civilian cases. Research has shown that 70 to 80 percent of veterans with PTSD treated in an inpatient setting have co-morbid substance use. Outpatient settings show rates of 50 to 60 percent of veterans with PTSD having co-morbid substance abuse. Civilian populations also show rates of co-morbidity of 50 to 60 percent. Whether people are treating their symptoms of PTSD with alcohol or drugs, thus causing this association, or whether co-morbid substance abuse decreases success of treatment of PTSD is still uncertain.

\section{Acknowledgements}

His clinical and research work with the Department of Defense and the Pentagon have led to new approaches for both the treatment and the prevention of post-traumatic stress disorder, including the present use of stress inoculation training for pre-deployed soldiers in all branches of the military. Dr. Singerman has served as a psychiatric expert witness for the last fifteen years. He has been involved in multiple cases with his legal colleagues, including cases in medical malpractice, personal injury, and disability litigation. His company website is burtonsingerman.com, and this website includes his full C.V. He presently conducts the majority of his psychiatric clinical work in a federally funded mental health clinic serving the Appalachian areas of South-western Pennsylvania.

\section{Conflicts of interests}

Author declares that there is no conflict of interest. 